\title{
Racial discrepancies in the association between paternal vs. maternal educational level and risk of low birthweight in Washington State
}

\author{
Christina Nicolaidis*1,2, Cynthia W Ko ${ }^{3}$, Somnath Saha1,2,4 and \\ Thomas D Koepsell 5
}

\begin{abstract}
Address: ${ }^{1}$ Department of Medicine, Oregon Health And Science University, Portland, Oregon, USA, ${ }^{2}$ Department of Public Health and Preventive Medicine, Oregon Health And Science University, Portland, Oregon, USA, ${ }^{3}$ Department of Medicine, University of Washington, Seattle,

Washington, USA, ${ }^{4}$ Section of General Internal Medicine, Portland Veterans Affairs Medical Center, Portland, Oregon, USA and ${ }^{5}$ Department of Epidemiology, University of Washington, Seattle, Washington, USA
\end{abstract}

Email: Christina Nicolaidis* - nicolaid@ohsu.edu; Cynthia W Ko - cwko@u.washington.edu; Somnath Saha - sahas@ohsu.edu; Thomas D Koepsell - koepsell@u.washington.edu

* Corresponding author

Published: 17 June 2004

BMC Pregnancy and Childbirth 2004, 4:10 doi:10.1 186/147I-2393-4-10

This article is available from: http://www.biomedcentral.com/I47/-2393/4/10

(C) 2004 Nicolaidis et al; licensee BioMed Central Ltd. This is an Open Access article: verbatim copying and redistribution of this article are permitted in all media for any purpose, provided this notice is preserved along with the article's original URL.
Received: 06 February 2004

Accepted: 17 June 2004

\begin{abstract}
Background: The role of paternal factors in determining the risk of adverse pregnancy outcomes has received less attention than maternal factors. Similarly, the interaction between the effects of race and socioeconomic status (SES) on pregnancy outcomes is not well known. Our objective was to assess the relative importance of paternal vs. maternal education in relation to risk of low birth weight (LBW) across different racial groups.

Methods: We conducted a retrospective population-based cohort study using Washington state birth certificate data from 1992 to $1996(n=264,789)$. We assessed the associations between maternal or paternal education and LBW, adjusting for demographic variables, health services factors, and maternal behavioral and obstetrical factors.

Results: Paternal educational level was independently associated with LBW after adjustment for race, maternal education, demographic characteristics, health services factors; and other maternal factors. We found an interaction between the race and maternal education on risk of LBW. In whites, maternal education was independently associated with LBW. However, in the remainder of the sample, maternal education had a minimal effect on LBW.

Conclusions: The degree of association between maternal education and LBW delivery was different in whites than in members of other racial groups. Paternal education was associated with LBW in both whites and non-whites. Further studies are needed to understand why maternal education may impact pregnancy outcomes differently depending on race and why paternal education may play a more important role than maternal education in some racial categories.
\end{abstract}

\section{Background}

Although overall neonatal and infant mortality rates have decreased substantially during this century, significant disparity still remains between socioeconomic and racial groups. In fact, the mortality rate gap between black and white infants has actually increased [1]. Several studies 
have examined the risk of adverse pregnancy outcomes such as low birthweight (LBW) and infant mortality in relation to maternal socioeconomic factors. Maternal socioeconomic characteristics known to affect the risk of adverse pregnancy outcomes include occupation, low income, and low educational level [2-4]. Younger age, parity ( 0 or $>4$ ), and being unmarried are also known risk factors [5]. However, the role of paternal factors in determining the risk of adverse pregnancy outcomes has received less attention than maternal factors. Similarly, the interaction between the effects of race and socioeconomic status (SES) on pregnancy outcomes is not well known. A comparison of the role of paternal vs. maternal socioeconomic factors is important as we attempt to understand economic and racial health disparities.

We undertook this study to assess the relative importance of paternal and maternal educational level in relation to risk of LBW delivery, while adjusting for demographic, behavioral, and health services factors. In this study, we used educational level as a proxy marker of SES and not necessarily as a direct cause of adverse pregnancy outcomes. Similarly, we use race as an important factor influencing socioeconomic status and cultural characteristics rather than an absolute biological distinction.

\section{Methods}

We conducted a retrospective population-based cohort study using Washington state birth certificate data. We began with all live singleton births in Washington state from 1992 through $1996(\mathrm{n}=362900)$. We excluded 784 births $(0.2 \%)$ where there were no data available about birthweight and 15030 births (4.1\%) in which either parent was age 18 or younger because we were concerned that educational level could not serve as a reasonable marker of SES for parents who were not old enough to have graduated from high school. In our primary analyses, we also excluded 82297 births (22.7\%) for which information was missing from the birth certificate about parental educational level, age, or race, (see table 1), leaving a sample of 264,789 births. Secondary analyses exploring the effect of missing data (described below) included all births with adult parents for which data are available on birthweight $(\mathrm{N}=347086)$.

The main outcome measure was LBW, defined as birthweight under 2500 grams. We chose LBW rather than premature delivery, since prior research has shown that data regarding birthweight are more accurate than estimated gestational age on birth certificates [6]. For our exposures, we used maternal and paternal educational level attained as documented on the birth certificate. We categorized years of education into 4 groups based on standard progression through the educational system $(<12$ years, 12 years, $13-15$ years, $\geq 16$ years). We chose educational level as opposed to other possible markers of SES, both because it is easier to accurately ascertain from birth certificate data, and because it allows us to make direct comparisons to findings from prior studies. Values outside of the plausible range in any of the study variables were recoded as missing.

In concordance with the coding scheme on Washington state birth certificates, parental race was classified into several mutually exclusive groups containing information on both race and ethnicity: white (non-Hispanic), black (non-Hispanic), Native American, Asian (including Pacific Islanders), and Hispanic. Thus, when we refer to "race" we, too, include information both on race and on whether or not the person was ethnically Hispanic. We did not have data about other ethnic groups. For the sake of clarity, we only present data separated by maternal race. Analyses looking at the effect of paternal race yielded relatively similar results.

We considered a number of other factors available from the birth certificates in order to better understand the relationship between educational level and LBW. We organized these data into 3 separate groups for the purposes of analysis. "Parental demographic factors" included maternal and paternal age (categorized as 19-24 years, 25-29 years, 30-34 years, and $\geq 35$ years), immigration status (foreign-born or not, as inferred from country of birth), and marital status (married or not). "Health services factors" included type of medical insurance used for this pregnancy (commercial/HMO, Medicaid, or uninsured), and late initiation of prenatal care (defined as initiation after the 1st trimester). "Maternal factors" included smoking during pregnancy (yes/no), alcohol use during pregnancy (yes/no), history of diabetes (yes/no), and parity $(0,1-3,4$ or more prior births). We chose to adjust for maternal factors last as we did not have similar data for the fathers, and thus wanted to look at the results both with and without adjustment for these factors.

Due to the high rate of incomplete data (see Table 1), we further explored the potential association between missing data and LBW. We speculated that missing data about fathers might be due either to factors leading to generally incomplete data (such as inability or lack of desire to accurately complete the birth certificate) or to the fact that the mother did not know the information about the father. In the former case, we would expect the birth certificate to have missing data about both the father and the mother, while in the latter case we would expect missing information only about the father. In either case, we expected that there would be higher LBW rates when paternal data were missing. In the former case, we hypothesized that factors associated with LBW - e.g., maternal illness - or maternal stress associated with adverse birth 
Table I: Numbers of births with missing data, by type of data.

\begin{tabular}{lc}
\hline Type of missing data & Births (\%) missing \\
\hline Maternal age & $296(0.1 \%)$ \\
Maternal race & $9966(2.9 \%)$ \\
Maternal education & $35670(10.3 \%)$ \\
Any maternal key data & $48307(13.9 \%)$ \\
Paternal age & $29997(8.6 \%)$ \\
Paternal race & $39772(11.5 \%)$ \\
Paternal education & $73421(21.15 \%)$ \\
Any paternal key data & $77737(22.4 \%)$ \\
Any key data & $82297(23.7 \%)$ \\
\hline
\end{tabular}

Numbers of births with missing data, by type of data. Note, some births had more than one type of missing data. "Key data" includes age, race and educational attainment.

outcomes - including LBW itself - might contribute to mothers' being unable or unwilling to complete the birth certificate. In the latter case, we speculated that mothers' not knowing key information about the father such as his race, age, or educational attainment might indicate that the mother was not close enough to the father to receive meaningful support from him. We therefore hypothesized that missing data about the father in otherwise complete birth certificates would be associated with greater risk of LBW at delivery, due to lack of paternal support during pregnancy. We thus conducted two secondary analyses. In one we assessed the association between missing paternal data and LBW at delivery after adjustment for missing maternal data (using missing maternal data as a proxy for completeness of birth certificate data). In the other, we assessed the association between missing paternal data and LBW at delivery in cases where maternal information was complete, stratifying by maternal race, and adjusting for all maternal information (maternal age, educational attainment, immigration, initiation of prenatal care, insurance status, diabetes, smoking, alcohol use and parity).

We report our results as crude and adjusted odds ratios which were derived using multiple logistic regression with Stata software (Version 6.0, Stata Corporation, College Station, Texas). For categorical variables, p-values correspond to the omnibus Wald test. We assessed the importance of effect modification using the significance of interaction terms in the logistic regression models.

\section{Results}

Amongst births with complete information on parental age, education, and race, $83 \%$ percent of mothers were white, 2.3\% black, 7.3\% Hispanic, and 5.9\% Asian. Eighty-two percent of fathers were white, 3.6\% black, $7.8 \%$ Hispanic, and $5.8 \%$ Asian. The mean ages of mothers and fathers were 28.3 years (range 19 to 56 years) and
30.7 years (range 19 to 75 years), respectively. Ninety percent of mothers were married. Prenatal care was initiated after the first trimester in 13\% of pregnancies. Thirteen percent of mothers smoked, and 1.3\% drank alcohol during the study pregnancy. Thirty-eight percent of mothers were nulliparous, while $4 \%$ reported 4 or more previous live births. The circumstances of births where at least one key piece of data on parental age, race, or education was missing differed greatly from those with complete data, with lower parental age and educational attainment, a lower proportion of married mothers, and greater racial diversity. (See table 2).

Educational level attained varied significantly by race for both mothers and fathers (Figure 1). A similar percentage of whites and blacks had less than a high school education, $(6.7 \%$ of white vs. $6.4 \%$ of black mothers; $9 \%$ of white vs. $4.7 \%$ of black fathers). However, whites and blacks differed in terms of higher education, with $28.4 \%$ of white vs. $17.7 \%$ of black mothers graduating from college, and $32.5 \%$ of white vs. $18.0 \%$ of black fathers graduating from college. The results for Hispanics were strikingly different, with $56.6 \%$ of mothers and $57.0 \%$ of fathers having less than a high school education, and only $5.3 \%$ of mothers and $5.5 \%$ of fathers graduating from college. Asian parents were the most likely to have graduated from college $(36.2 \%$ of mothers and $42.2 \%$ of fathers, respectively). Native American parents attained educational levels intermediate to the other groups described here.

The unadjusted prevalence of LBW also varied by maternal race. Overall, blacks were over twice as likely to have a LBW infant than whites ( $7.2 \%$ vs. $3.3 \%$ for infants born to white vs. black mothers, respectively.). Other racial groups had overall prevalences of LBW closer to that of whites, despite the educational characteristics described 
Table 2: Demographic characteristics

\begin{tabular}{lcc}
\hline & No missing data (N = 264789) & Any missing data (N = 82297) \\
\hline Maternal Age: mean (std)* & $28.3(5.4)$ & $26.8(5.7)$ \\
Maternal Education: mean (std)* & $11.8(2.7)$ \\
Maternal Race: (N, \%)* & & \\
White & $219455(82.9 \%)$ & $46483(64.3 \%)$ \\
Black & $6078(2.3 \%)$ & $5647(7.8 \%)$ \\
Asian \& Pacific Islander & $16072(6.1 \%)$ & $6378(8.8 \%)$ \\
Hispanic & $19271(7.3 \%)$ & $11081(15.3 \%)$ \\
Native American \& other & $3913(1.5 \%)$ & $2742(3.8 \%)$ \\
Paternal Age: mean (std)* & $30.7(6.1)$ & $30.4(6.7)$ \\
Paternal Education: mean (std)* & $13.4(2.7)$ & $12.8(3.9)$ \\
Paternal Race: (N, \%) & & \\
White & $217539(82.2 \%)$ & $25572(60.1 \%)$ \\
Black & $9615(3.6 \%)$ & $3644(8.6 \%)$ \\
Asian \& Pacific Islander & $13184(5.0 \%)$ & $4098(9.6 \%)$ \\
Hispanic & $20625(7.8 \%)$ & $8267(19.4 \%)$ \\
Native American \& other & $3826(1.4 \%)$ & $944(2.2 \%)$ \\
Married mother: (N, \%) & $238092(89.9 \%)$ & $37213(45.2 \%)$ \\
LBW at delivery: (N, \%) & $9337(3.5 \%)$ & $5202(6.3 \%)$
\end{tabular}

Demographic characteristics and rate of low birthweight at delivery for births with and without missing data. $\mathrm{N}=$ number of births; std = standard deviation. LBW $=$ low birthweight. $* \mathrm{P}<0.001$

above. The unadjusted prevalence of LBW was $3.9 \%$ in Native Americans, $4.3 \%$ in Asians, and 3.8\% in Hispanics.

As expected, maternal educational level alone was significantly associated with LBW in all races combined (Table $3)$. Using the group in which the mother had graduated from college ( $\geq 16$ years of education) as the reference group, the group in which the mother had not graduated from high school had an unadjusted odds ratio of 1.59 (95\% confidence interval (CI) 1.47, 1.70) for risk of LBW. Similarly, the unadjusted odds ratio for LBW comparing infants whose fathers had not graduated from high school to those whose fathers had graduated from college was $1.58(95 \%$ CI $1.47,1.69)$ for all races combined.

The effect of maternal educational level on risk of LBW varied by race (significance of interaction term: p < 0.0001 ). When analyzing each racial group separately, the effect of maternal education on risk of LBW was considerably different in births where the mother was white (OR 1.99) than it was in any of the other racial groups (OR ranged between 0.89-1.05). We, thus, reanalyzed the data excluding whites. In births where the mother was not white, the rate of LBW varied by race, but the effect of education on rate of LBW did not (significance of interaction term: $p=0.6$ ). Hence, results where the mother was not white are presented separately by race, but are also presented in the aggregate after adjustment for confounding by racial category. When looking at white mothers separately, the odds ratio for LBW in births where the mother had not graduated from high school went up to 1.99 $(95 \%$ CI 1.82, 2.17) and the odds ratio for LBW in births were the father had not graduated from high school went up to 1.88 (95\% CI $1.72,2.06)$. The unadjusted odds ratios for either maternal or paternal education were close to 1.0 for each of the other racial groups (Table 3 ).

Table 4 displays the effects of adjusting for covariates on odds ratios for LBW in births where the parent in question had less than a high school education as compared to those where that parent had graduated from college, stratified by white vs. nonwhite maternal race. Among whites, the odds ratios for both maternal and paternal educational level became progressively smaller with adjustment first for the other parent's educational level, then adding parental demographic factors (parental age, immigration status, and marital status), then adding health services factors (insurance type and initiation of prenatal care after the first trimester), and finally adding maternal factors (smoking during pregnancy, alcohol use during pregnancy, history of diabetes, and parity). After adjustment for all variables mentioned, the odds ratio in whites was $1.5(95 \%$ CI 1.3, 1.7) for maternal educational level and $1.3(95 \%$ CI 1.2, 1.5) for paternal educational level. It should be noted that, in every model tested, both maternal and paternal educational level were independ- 

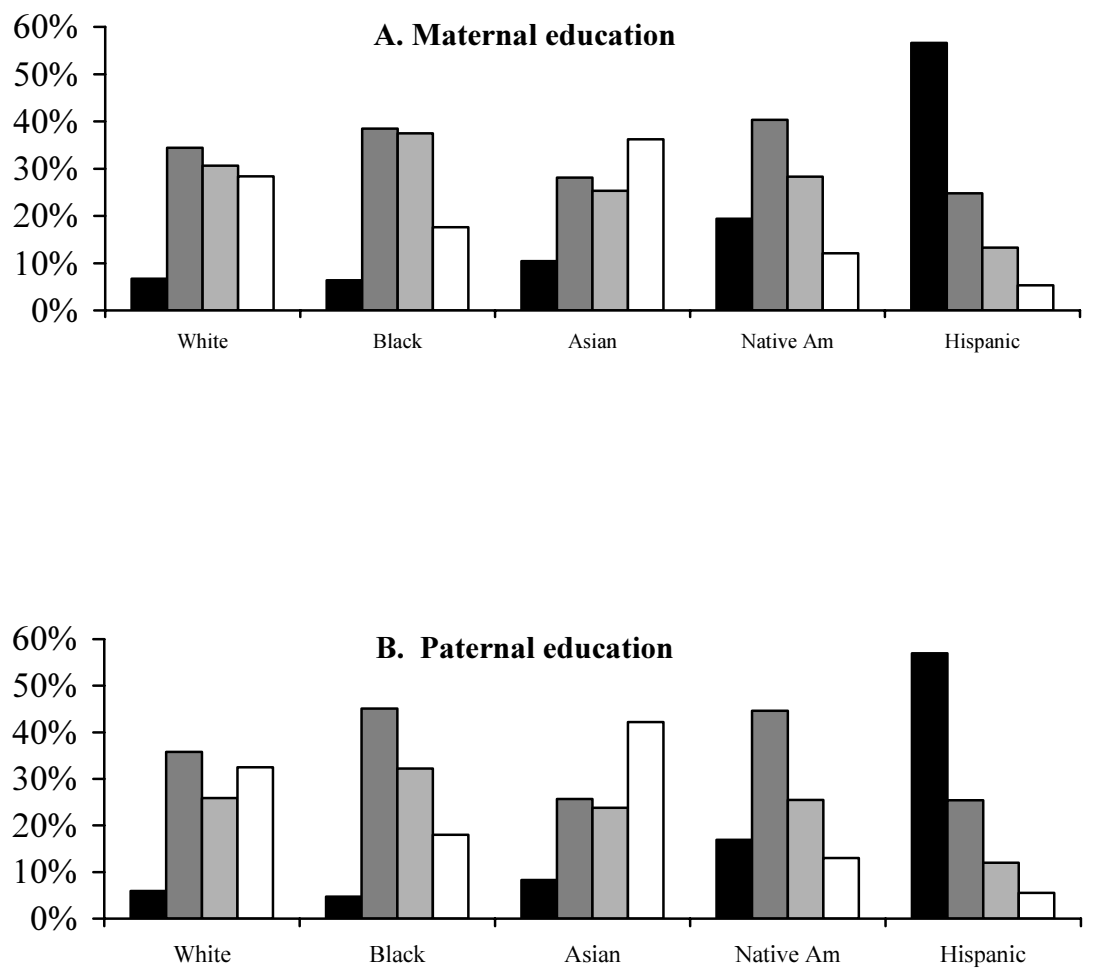

$\square<12$ years $\square 12$ years $\square 13-15$ years $\square>=16$ years

Figure I

Parental education by race. A. Maternal education. B. Paternal education. Percent of infants within each racial group born to parents with $<12,12,13-15$, or $>=16$ years of education.

ently associated with the risk of LBW among white mothers, an association which was ultimately not explained by any of the other factors we considered.

In births where the mother was not white, the relationship was less straightforward (Table 4). Maternal educational level did not appear to be associated with risk of LBW in any of the models, with non-significant odds ratios close to one both before and after adjustment for other available variables. In contrast, paternal educational level was significantly associated with the risk of LBW, after adjustment for maternal educational level, parental demographics, and health services factors. It remained so after adjustment for maternal behavioral and obstetrical factors. In the final adjusted model, the odds ratio for LBW in non-white births where the father had less than a high school education, as compared to those where the father had graduated from college, was 1.4 (95\% CI 1.1, 1.8), a degree of association similar to that seen in whites. Finally, we developed a logistic regression model which included all potential confounders considered. For this analysis, we again separated births where the mother was white from the rest of the sample (Table 5). In whites, maternal educational level, paternal educational level, maternal age, insurance status, maternal smoking, and parity were independently associated with the risk of LBW. For births where the mother was not white, paternal educational level, paternal race, maternal age, paternal age, marital status, maternal smoking, maternal alcohol use, and parity were independently associated with LBW. Maternal educational level was not significantly associated with LBW in non-whites. Secondary analyses where non-significant variables were eliminated from the model or where the sample was restricted to married couples yielded similar results (data not shown). 
Table 3: Unadjusted prevalence of low birthweight by maternal or paternal educational level amongst different racial groups

\begin{tabular}{|c|c|c|c|c|c|c|c|c|c|c|c|c|}
\hline & \multicolumn{2}{|c|}{ All Races } & \multicolumn{2}{|c|}{ Whites } & \multicolumn{2}{|c|}{ Blacks } & \multicolumn{2}{|c|}{ Asians } & \multicolumn{2}{|c|}{ Hispanics } & \multicolumn{2}{|c|}{ All Non-White } \\
\hline & OR* & $95 \% \mathrm{Cl} \dagger$ & $\mathrm{OR} *$ & $95 \% \mathrm{Cl} \dagger$ & OR* & $95 \% \mathrm{Cl} \dagger$ & OR* & $95 \% \mathrm{Cl} \dagger$ & OR* & $95 \% \mathrm{Cl} \dagger$ & OR* & $95 \% \mathrm{Cl} \dagger$ \\
\hline \multicolumn{13}{|l|}{$\begin{array}{l}\text { Maternal } \\
\text { Education } \ddagger\end{array}$} \\
\hline$<12$ & 1.59 & $1.47-1.70$ & 1.99 & $1.82-2.17$ & 0.90 & $0.56-1.45$ & 1.05 & $0.80-1.38$ & 0.99 & $0.70-1.39$ & 0.96 & $0.82-1.12$ \\
\hline 12 & 1.29 & $1.23-1.37$ & 1.33 & $1.25-1.42$ & 1.10 & $0.83-1.46$ & 1.06 & $0.87-1.28$ & 1.12 & $0.78-1.60$ & 1.06 & $0.92-1.21$ \\
\hline $13-15$ & 1.16 & $1.09-1.23$ & 1.15 & $1.08-1.23$ & 1.08 & $0.81-1.43$ & 1.09 & $0.90-1.33$ & 1.33 & $0.92-1.94$ & 1.12 & $0.98-1.29$ \\
\hline$>=16$ & 1.00 & Referent & 1.00 & Referent & 1.00 & Referent & 1.00 & Referent & 1.00 & Referent & 1.0 & Referent \\
\hline \multicolumn{13}{|l|}{$\begin{array}{l}\text { Paternal } \\
\text { Education } \neq\end{array}$} \\
\hline$<12$ & 1.58 & $1.47-1.69$ & 1.88 & $1.72-2.06$ & 0.96 & $0.58-1.57$ & 1.23 & $0.91-1.66$ & 0.97 & $0.72-|.3|$ & 1.06 & $0.91-1.24$ \\
\hline 12 & 1.37 & $1.30-1.45$ & 1.41 & $1.32-1.50$ & 0.96 & $0.74-1.25$ & 1.10 & $0.91-1.33$ & 1.11 & $0.81-1.52$ & 1.10 & $0.97-1.26$ \\
\hline $13-15$ & 1.21 & $1.14-1.28$ & 1.23 & $|| 5-.|.3|$ & 0.84 & $0.63-1.11$ & 1.19 & $0.98-1.45$ & 1.00 & $0.70-1.43$ & 1.04 & $0.90-1.14$ \\
\hline$>=16$ & 1.00 & Referent & 1.00 & Referent & 1.00 & Referent & 1.0 & Referent & 1.00 & Referent & 1.00 & Referent \\
\hline
\end{tabular}

Columns are separated by maternal race. "Non-white" includes infants whose mother was black, Asian, Hispanic, Native American or "other". Odds ratios for non-whites were adjusted for maternal race. * OR: odds ratio. $\dagger 95 \% \mathrm{Cl}$ : $95 \%$ confidence interval. $\ddagger$ Years of education completed.

Table 4: Association between risk of low birthweight and maternal vs. paternal education after serial adjustments for other potential confounders.

\begin{tabular}{|c|c|c|}
\hline Adjustments & Maternal Education OR $*(95 \% \mathrm{Cl} \dagger)$ & Paternal Education OR $*(95 \% \mathrm{Cl} \dagger)$ \\
\hline \multicolumn{3}{|l|}{ Whites } \\
\hline none & $2.0(1.8-2.2)$ & $1.9(1.7-2.1)$ \\
\hline other parent's education & $1.6(1.4-1.8)$ & $1.5(1.4-1.7)$ \\
\hline above + parental demographics & $1.6(1.5-1.8)$ & $1.5(1.4-1.7)$ \\
\hline above + health services factors & $1.5(1.4-1.7)$ & $1.4(1.3-1.6)$ \\
\hline above + maternal factors & $1.5(1.3-1.7)$ & $1.3(1.2-1.5)$ \\
\hline \multicolumn{3}{|l|}{ Non-whites } \\
\hline maternal and paternal race & $\mathrm{I} .0(0.8-\mathrm{I} . \mathrm{I})$ & I.I (0.9-I.2) \\
\hline above + other parent's education & $0.9(0.7-1.1)$ & $1.2(1.0-1.5)$ \\
\hline above + parental demographics & $0.9(0.7-1.1)$ & $1.2(0.9-1.3)$ \\
\hline above + health services factors & $0.8(0.6-1.0)$ & $1.3(1.1-1.7)$ \\
\hline above + maternal factors & $0.8(0.7-I . I)$ & $1.4(1.1-1.8)$ \\
\hline
\end{tabular}

All odds ratios compare the group where the parent had not graduated from high school to group where the parent had graduated from college. Parental demographics includes parental ages, immigration status, and marital status. Health services factors include maternal insurance status and trimester of initiation of prenatal care. Maternal factors include parity, smoking, diabetes, and alcohol use. * OR: Odds ratio. † $95 \% \mathrm{Cl}: 95 \%$ confidence interval.

Table 6 shows the results of the full model when each race is analyzed separately. Adjusted odds ratios for the association between maternal education and LBW at delivery were consistently close to 1.0 for all races other than whites. Adjusted odds ratios for the association between paternal education and LBW at delivery ranged between 1.2 and 1.5 , though they do not reach statistical significance in the nonwhite groups unless aggregated together.

Births with missing information on either parent had a higher risk of LBW at delivery than those with complete information (unadjusted OR 1.84; CI 1.78-1.91). The effect of missing paternal information on risk of LBW at delivery remained significant even after adjustment for whether or not there was any missing maternal information (OR 1.92, CI 1.86-2.00) or for the number of pieces of missing maternal information (OR 1.68, CI 1.62$1.75)$. In births with complete maternal information, the odds of LBW at delivery was 1.36 (CI 1.28-1.46) for births with missing paternal information, after adjustment for all available maternal information. When separating analyses by maternal race, the effect of missing paternal information remained significant for Whites (OR 1.32, CI 1.22-1.44), Blacks (OR 1.57; CI 1.17-2.09), Asians (OR 1.72; CI 1.29-2.29), and Hispanics (OR 1.44; CI 1.171.77). 
Table 5: Adjusted odds ratios for risk of low birthweight using full model

\begin{tabular}{|c|c|c|c|c|}
\hline & \multicolumn{2}{|c|}{ Mother White } & \multicolumn{2}{|c|}{ Mother Non-White } \\
\hline & OR* & $\mathrm{P}$-value & OR* & $\mathrm{p}$-value \\
\hline Maternal Education $\dagger$ & & $<0.0001$ & & 0.13 \\
\hline$<12$ & 1.49 & & 0.85 & \\
\hline 12 & 1.17 & & 0.95 & \\
\hline $13-15$ & 1.11 & & 1.08 & \\
\hline$>=16$ & referent & & referent & \\
\hline Paternal Education $\dagger$ & & 0.0001 & & .04 \\
\hline$<12$ & 1.30 & & 1.40 & \\
\hline 12 & 1.20 & & 1.25 & \\
\hline $13-15$ & 1.10 & & 1.11 & \\
\hline$>=16$ & referent & & referent & \\
\hline Maternal Race & & $\mathrm{n} / \mathrm{a}$ & & 0.08 \\
\hline White & $\mathrm{n} / \mathrm{a}$ & & $\mathrm{n} / \mathrm{a}$ & \\
\hline Hispanic & $\mathrm{n} / \mathrm{a}$ & & referent & \\
\hline Black & $\mathrm{n} / \mathrm{a}$ & & 1.43 & \\
\hline Asian or Pacific Islander & $\mathrm{n} / \mathrm{a}$ & & 1.28 & \\
\hline Native American or Other & $\mathrm{n} / \mathrm{a}$ & & 1.11 & \\
\hline Paternal Race & & 0.90 & & 0.001 \\
\hline White & Referent & & 0.73 & \\
\hline Hispanic & 0.94 & & Referent & \\
\hline Black & 0.99 & & 1.21 & \\
\hline Asian or Pacific Islander & 1.00 & & 0.87 & \\
\hline Native American or Other & 0.89 & & 1.34 & \\
\hline Maternal Age (years) & & $<.0001$ & & 0.004 \\
\hline 19-24 & referent & & referent & \\
\hline $25-29$ & 1.17 & & 1.17 & \\
\hline $30-34$ & 1.25 & & 1.34 & \\
\hline$>=35$ & 1.61 & & 1.53 & \\
\hline Paternal Age (years) & & 0.4 & & 0.048 \\
\hline 19-24 & referent & & referent & \\
\hline $25-29$ & 1.09 & & 0.75 & \\
\hline $30-34$ & 1.09 & & 0.67 & \\
\hline$>=35$ & 1.09 & & 0.82 & \\
\hline Marital Status & & 0.05 & & 0.035 \\
\hline yes & 1.09 & & 1.20 & \\
\hline no & referent & & referent & \\
\hline Insurance & & $<.0001$ & & 0.42 \\
\hline Commercial / HMO & referent & & referent & \\
\hline Medicaid & 1.25 & & 0.93 & \\
\hline Uninsured & 0.94 & & 1.09 & \\
\hline Maternal Smoking & & $<.0001$ & & $<.0001$ \\
\hline yes & 2.10 & & 1.74 & \\
\hline no & referent & & referent & \\
\hline Maternal Alcohol Use & & 0.13 & & 0.01 \\
\hline yes & 1.17 & & 1.03 & \\
\hline no & referent & & referent & \\
\hline Parity (number of prior & & $<.0001$ & & $<.0001$ \\
\hline 0 & referent & & referent & \\
\hline $1-3$ & 0.54 & & 0.65 & \\
\hline$>=4$ & 0.57 & & 0.71 & \\
\hline
\end{tabular}

White denotes infants whose mother was white. Non-white denotes infants whose mother was Black, Asian, Hispanic, Native American, or Other. For categorical variables, $\mathrm{p}$-values correspond to the significance of the omnibus Wald test for the entire variable, not a specific category. Model also adjusts for immigration status, initiation of prenatal care after the Ist trimester, and maternal diabetes. * OR: adjusted odds ratio. n/a: not applicable. †Years of education completed. 
Table 6: Association between maternal vs. paternal educational attainment and LBW at delivery, after adjustment for demographic factors, health services factors, and maternal factors, by maternal race.

\begin{tabular}{|c|c|c|c|c|}
\hline \multirow[t]{2}{*}{ Maternal Race } & \multicolumn{2}{|c|}{ Mother with less than high school education } & \multicolumn{2}{|c|}{ Father with less than high school education } \\
\hline & Adj. OR & $95 \% \mathrm{Cl}$ & Adj. OR & $95 \% \mathrm{Cl}$ \\
\hline White & 1.5 & I.3-1.7 & 1.3 & $1.1-1.5$ \\
\hline Black & 0.8 & $0.4-1.7$ & 1.2 & $0.6-2.4$ \\
\hline Asian & 0.9 & $0.6-1.4$ & 1.5 & $1.0-2.4$ \\
\hline Hispanic & 0.9 & $0.5-1.5$ & 1.5 & $1.0-2.5$ \\
\hline All non-white & 0.8 & $0.7-1.0$ & 1.4 & $1.1-1.8$ \\
\hline
\end{tabular}

Association between maternal vs. paternal educational attainment and low birthweight at delivery, by maternal race, after adjustment for demographic factors (maternal and paternal age, marital status, and immigration), health services factors (type of insurance and late initiation of prenatal care), and maternal factors (smoking, alcohol use, diabetes, and parity). Adjusted odds ratios compare the group where either parent had not completed high school to the group where the parent had graduated from college. Adj. OR: adjusted odds ratio; Cl: $95 \%$ confidence interval.

\section{Discussion and conclusions}

Our study found the magnitude of the association between parental educational level and rate of low birthweight was different in births where the mother was white than in it was for members of other racial groups. In whites, low maternal and paternal educational levels were independently associated with a higher risk of LBW, even after adjustment for available demographic, health services, and maternal factors. In births where the mother was not white, maternal educational level appeared to have little or no effect on risk of LBW. However, low paternal educational level was independently associated with a higher risk of LBW after adjustment for maternal educational level, race, and other demographic and health services variables. The effect of paternal educational level remained significant after adjustment for maternal factors.

An association between SES and health is widely recognized. People of lower SES have worse health status, with higher mortality rates for all causes of death, higher prevalence of chronic diseases, and higher measures of disability [7]. We found that maternal educational level was associated with the risk of LBW in whites, but not in other racial groups. Prior literature has noted that the discrepancy in the risk of adverse pregnancy outcomes between blacks and whites increased at higher levels of education $[1,8]$. Also, prior studies have found relatively low rates of adverse pregnancy outcomes in Hispanics despite socioeconomic disadvantage $[8,9]$. Our findings suggests that maternal educational level may be less important in influencing the risk of adverse pregnancy outcomes in blacks or Hispanics than it is in whites. Our study contributes the additional finding that paternal educational level is significantly associated with LBW regardless of race.
Other studies in the United States have examined the relationship between paternal characteristics and utilization of prenatal care, LBW, small-for-gestational-age status, and premature delivery. Parker, et al., found that both maternal and paternal educational level were associated with low birthweight and prematurity [10]. D'Ascoli, et al., found that higher paternal educational level was associated with early initiation of prenatal care, even after adjusting for maternal educational level [11]. Another study found that paternal educational level and race were independent risk factors for LBW [12]. Here, the effects of maternal and paternal educational level were similar, and no interactions were found between the two measures. However, the latter two studies did not examine the effects of parental educational level separately by race. Also, in most studies, minority groups other than blacks were not studied.

Though we can only speculate about possible explanations for our findings, doing so may allow us to generate new hypotheses to be tested in future research. One possible explanation of these findings may relate to the variable impact of educational attainment across racial groups and between men and women. For example, the increase in income and social status that typically accompanies advanced educational attainment may be less substantial for nonwhites than whites, particularly among women [8]. If this were the case in our population, there may have been less variability across educational levels, among nonwhite as compared to white women, in financial resources and other protective factors that appear to accompany higher SES. This would tend to minimize the association between educational level and the risk of LBW in nonwhite women. Another possibility is that the mix of social, cultural, and biological factors conferring protective (among Hispanics) or harmful (among African-Americans) effects on birth outcomes among nonwhite women 
are so influential that the effect of maternal education is rendered negligible.

Our study has a number of important limitations. First, we had to rely on the accuracy and completeness of birth certificate data. In fact, we excluded over $20 \%$ of birth certificates due to missing data on parental education, age, or race. Births with missing parental information appear to be significantly different from those with complete information. The fact that there were higher rates of LBW at delivery for births with incomplete data could be due to parents' having less ability or interest in completing the birth certificate form when suffering the stress of having just delivered a LBW infant. Moreover, SES and cultural factors may influence a parent's ability or desire to accurately complete the form, as suggested by the lower educational attainment and greater racial and ethnic diversity seen in the group with incomplete birth certificate data. However, missing paternal information, in the setting of complete maternal information, may be due to the mother's not knowing who the father is, not wishing to list him on the birth certificate, or not knowing him well enough to answer the questions about his race, educational attainment, or age. In any of these cases, one would assume that the mother may not be receiving significant support from the father. We found that missing paternal information was significantly associated with risk of LBW at delivery, even after adjustment for the level of completeness of maternal information. Moreover, the effect of missing paternal information remained significant after adjustment of all available maternal information, even when analyzing each race separately. These findings are consistent with our hypothesis that paternal support plays an important role in the risk of LBW at delivery in all the racial groups we studied.

Second, we used parental educational level as a proxy measure of SES, since it is easily measured and obtained from birth certificates. However, SES is a difficult construct to quantify, and educational level is only one of the many variables that may influence SES. Educational attainment cannot serve as a proxy for teen parents as they have not had a chance to attain higher levels of education. Our results, thus, cannot be generalized to parents under the age of 19. Income, another indication of SES, is not available from Washington state birth certificates. Also, occupational status, although available on Washington state birth certificates, is difficult to classify into meaningful categories for these purposes. The complex interaction between these and other variables in defining one's SES is difficult to quantify for purposes of this type of study, and thus, our use of educational level as a proxy for SES likely does not fully capture this concept. Third, even using 5 years of birth certificate data, we had an inadequate sample size to assess rare outcomes such as infant mortality, and thus had to rely on the intermediate outcome of LBW. Fourth, we cannot infer a causal association between parental educational level and the risk of LBW from these observational data.

Lastly, we conducted this study using only data from the state of Washington - a state which has relatively small proportions of racial minorities as compared to other parts of the U.S. This may have limited our ability to detect significant differences between each of the nonwhite racial groups. For example, though the effect of maternal education on risk of LBW did not vary by race once whites were excluded from the analysis, it is possible that effect modification by race does exist, even in the other racial groups. Not having found evidence of such, we chose to group the other racial categories together to improve our statistical power to detect any effect of parental education on LBW. Analyses looking at each race separately yielded a similar pattern, but did not reach statistical significance, presumably due to low numbers. We do not wish to imply that members of the different racial categories are similar in regards to birth outcomes, SES, or the many cultural factors that can influence both. The grouping simply results from the finding that the magnitude of association between educational level and LBW is similar amongst all racial groups studied other than whites. All analyses using this grouping still adjust for race.

These limitations notwithstanding, we believe our study adds important information about the effect of paternal SES in the risk of LBW in different races. Studies examining maternal SES have focused only on the relationship between the mother and the fetus to explain pregnancy outcomes. The additional independent association seen between paternal educational level and pregnancy outcomes may speak to the importance of looking beyond the maternal-fetal dyad to the world surrounding a pregnancy. For example, family and community support may play an important role in determining pregnancy outcomes. Paternal educational level may be a marker for the father's ability to provide the mother such support during her pregnancy or to shield her from societal stressors. Further studies looking at the balance between societal stressors and support systems available to a mother during pregnancy may not only help explain our findings about paternal educational levels but may also shed light on the discrepancies amongst different racial and ethnic groups.

We conclude that paternal educational level is associated with the risk of LBW regardless of race, but that maternal educational level seems only to be a significant factor in whites. The reason for the different degree of association in whites than in members of other racial groups is not clear and deserves further examination. This study has 
important implications. First, obtaining information on paternal educational levels may improve our ability to identify groups at highest risk for LBW, in both whites and nonwhites. Identifying these groups may allow the development and implementation of targeted interventions to reduce the risk of such adverse pregnancy outcomes. Second, the assumption that maternal SES strongly relates to pregnancy outcomes may only be true for whites. This relationship may be significantly different for members of other racial groups. Studies looking at risk factors and mechanisms for LBW should conduct race-stratified analyses, or at least should routinely examine the possible interaction between race and education. Studies using national vital statistic data are needed in order to confirm our results in each separate racial category. If confirmed, this racial discrepancy between the relative effect of maternal vs. paternal SES may prove to be an interesting clue to the role of differences in family and community support systems, income, and discrimination in birth outcome disparities. Further studies using data beyond what is available on birth certificates will be needed to better understand these complex relationships.

\section{Competing interests}

None declared.

\section{Author contributions}

$\mathrm{CN}$ conceptualized the study, conducted the analyses, and wrote the manuscript.

CK participated in the conceptualization of the study, the initial analyses, and the initial writing of the manuscript.

SS made suggestions regarding secondary analyses and helped revise the manuscript.

TK mentored CN and CK in all aspects of study design and analysis and edited the manuscript.

All authors have read and approved the final version of the manuscript.

\section{Acknowledgments}

We acknowledge the assistance of Drs. Cathy Critchlow, Beth Mueller, and Tom Vaughan with the initial stages of this project, and the help of Bill O'Brien with computer programming. We also acknowledge the Washington State Department of Health for allowing access to the birth certificate data. Drs. Nicolaidis and Ko were Robert Wood Johnson Clinical Scholars at the time of this work. Dr. Nicolaidis is currently the recipient of a Robert Wood Johnson Depression In Primary Care Leadership Award. Dr. Saha is the recipient of a Research Career Development award from the Department of Veterans Affairs Health Services Research \& Development Service and is a Robert Wood Johnson Foundation Generalist Physician Faculty Scholar. Funding was provided by the Robert Wood Johnson Foundation via the Clinical Scholars Program. The views expressed are those of the authors alone and do not necessarily reflect those of the Robert Wood Johnson Foundation.

\section{References}

I. Schoendorf K, Hogue C, Kleinman J, Rowley D: Mortality among infants of black as compared to white college-educated parents. N Engl J Med 1992, 326:1522-6.

2. McCormick M: The contribution of low birth weight to infant mortality and childhood morbidity. N Engl J Med 1985, 3 I 2:82-90.

3. Rush D, Cassano P: Relationship of cigarette smoking and social class to birth weight and perinatal mortality among all births in Britain 5-II April 1970. J Epid Commun Health 1983 , 37:249-55.

4. Ericson A, Eriksson M, Jetterstrom R: Analysis of perinatal mortality rate in the Stockholm area. Acta Pediatrica Scan Supple 1979, 275:35-40.

5. Luke B, Williams C, Minogue J, Keith L: The Changing pattern of infant mortality in the US: the role of prenatal factors and thier obstetrical implications. Int J Gynecol Obstet 1993, 40:199-2/2.

6. David R: The quality and completeness of birthweight and gestational age data in computerized data files. Am J Public Health 1980, 70:964-973.

7. Whitehead $M$ : The health divide: inequalities in health in the 1980 s. London: The Health Education Council; 1987.

8. Krieger N, Rowley D, Herman A, Avery B, Phillips M: Racism, sexism, and social class: implications for studies of health, disease, and well-being. Am J Prev Med 1993, 9(6 Suppl):82-122.

9. Fuentes-Aflick E, Hessol NA, Perez-Stable El: Testing the epidemiologic paradox of low birth weight in Latinos. Arch Pediatr Adolesc Med 1999, 153:147-153.

10. Parker JD, Schoendorf KC, Kiely JL: Associations between measures of socioeconomic status and low birth weight, small for gestational age, and premature delivery in the United States. Ann Epidemiol 1994, 4:27I-278.

II. D'Ascoli PT, Alexander GR, Petersen DJ, Kogan MD: Parental factors influencing patterns of prenatal care utilization. J Perinatol 1997, 17:283-287.

12. Parker JD, Schoendorf KC: Influence of paternal characterisitcs on the risk of low birth weight. Am J Epidemiol 1992, 136:399-407.

\section{Pre-publication history}

The pre-publication history for this paper can be accessed here:

http://www.biomedcentral.com/1471-2393/4/10/prepub
Publish with Bio Med Central and every scientist can read your work free of charge

"BioMed Central will be the most significant development for disseminating the results of biomedical research in our lifetime. "

Sir Paul Nurse, Cancer Research UK

Your research papers will be:

- available free of charge to the entire biomedical community

- peer reviewed and published immediately upon acceptance

- cited in PubMed and archived on PubMed Central

- yours - you keep the copyright
BioMedcentral 